\title{
Isolation and characterization of pre-mRNA splicing mutants of Saccharomyces cerevisiae
}

\author{
Usha Vijayraghavan, Mahshid Company, and John Abelson \\ Division of Biology, 147-75, California Institute of Technology, Pasadena, California 91125 USA
}

\begin{abstract}
In this study we report the isolation of temperature-sensitive mutants that affect pre-mRNA splicing. A bank of $\sim 1000$ temperature-sensitive Saccharomyces cerevisiae strains was generated and screened on RNA gel blots by hybridization with an actin intron probe. We isolated 16 mutants defining 11 new complementation groups prp(rna)17-prp(rna)27 with four phenotypic classes of mutants and 21 mutants in the prp2-prp11 complementation groups (formerly rna2-rna11). The majority of the complementation groups share a phenotype of pre-mRNA accumulation, seen in all of the prp(rna)2-prp(rna)11 mutants. Three novel classes of mutants were isolated in this study. One class, consisting of two complementation groups, exhibits an accumulation of the lariat intermediate of splicing, with no change in the levels of pre-mRNA. The second class, also represented by two complementation groups, shows an accumulation of the intron released after splicing. The third novel class, comprising one complementation group, accumulates both pre-mRNA and the released intron. All mutants isolated were recessive for the splicing phenotype. Only 2 of the 11 complementation groups, although recessive, were not temperature sensitive. This study, together with previous isolation of the prp(rna)2-prp(rna)11 groups and the spliceosomal snRNAs, puts at least 26 gene products involved directly or indirectly in pre-mRNA splicing.
\end{abstract}

[Key Words: Saccharomyces cerevisiae; temperature-sensitive mutants; prp(rna)17-27]

Received April 24, 1989; revised version accepted June 14, 1989.

The precise removal of the intron from nuclear premRNA is an essential process in eukaryotic gene expression. The splicing reaction takes place in a complex particle termed the spliceosome (Brody and Abelson 1985; Frendewey and Keller 1985; Grabowski et al. 1985). The function of the spliceosome in nuclear pre-mRNA splicing is to align the splice sites and to catalyze the two-step splicing reaction. Therefore, to understand mRNA splicing, it will not only be necessary to elucidate the components and their functions but also the pathway of assembly of the spliceosome.

The process of pre-mRNA splicing appears to be very similar in the yeast and the mammalian systems, but a particular advantage to the study of splicing in yeast is the facility with which a genetic approach can be applied to this problem. Not only can mutants defective in splicing be used to enumerate the components and their interactions but they can be useful in delineating steps in the assembly of the spliceosome. Before the process of pre-mRNA splicing was discovered, a set of temperature-sensitive mutants in RNA synthesis was found that defined the RNA2-RNA11 genes (Hartwell 1967). Surprisingly, all of these mutants are defective in mRNA splicing (for review, see Warner 1987; Vijayraghavan and Abelson 1989). By general consensus among the community of researchers working on RNA processing in yeast and the yeast genetic stock center, these mutants will henceforth be called pre-RNA processing (prp) mutants. In vitro biochemical experiments demonstrated the direct participation of many of the PRP2-PRP11 gene products in the pre-mRNA splicing process (Lustig et al. 1986). Splicing activity in extracts prepared from the prp2, prp3, prp4, prp5, prp7, prp8, and prp11 strains are heat sensitive in vitro. The loss of activity was shown to be due to the inactivation of an exchangeable component in each case by in vitro complementation of pairs of inactivated extracts. Except for PRP2, the PRP gene products $3,4,5,7,8$, and 11 are required for the assembly of the spliceosome (Lin et al. 1987). Molecular characterization of some of the cloned PRP genes has indicated that they code for proteins (Last et al. 1984; Lee et al. 1984; Soltyk et al. 1984; Jackson et al. 1988). Antibodies directed against PRP8 fusion proteins inhibit in vitro splicing reactions, and these antibodies have been used to detect an association of the PRP8 protein and the yeast U5 small nuclear RNA (snRNA) (Lossky et al. 1987). In the case of $P R P 11$, the ${ }^{35}$ S-labeled protein was shown to associate with the $40 \mathrm{~S}$ spliceosome and also to associate with a 30 S complex (Chang et al. 1988). The role of the other PRP gene products are being investigated in several laboratories. Apart from the identification of the $P R P$ genes, the five spliceosomal U snRNAs 
of yeast have been identified and are single-copy genes (Guthrie and Patterson 1988).

A combination of genetic and biochemical techniques therefore is proving to be extremely useful in the identification of pre-mRNA splicing factors. About $5 \%$ of the original Hartwell collection of 400 temperature-sensitive lethal mutants fell into the $P R P$ class. However, this collection of splicing mutants is not complete because there were only single alleles in many of the complementation groups. The need for a number of transacting factors in pre-mRNA splicing also is apparent from the complexity of the spliceosome and the premRNA splicing process. To isolate more mutants defective in pre-mRNA splicing, we constructed a bank of temperature-sensitive yeast strains by chemical mutagenesis. The mutant bank has been screened directly for pre-mRNA processing defects by a Northern blot assay. Genetic characterization, including complementation and segregation analysis, has been conducted for all of the candidates. The screen of $\sim 1000$ temperature-sensitive lethal mutants resulted in the identification of several new complementation groups. Some of these new complementation groups show novel phenotypes, implying a role for these gene products in various steps of the splicing pathway.

\section{Results}

Generation of a bank of temperature-sensitive lethal mutants in Saccharomyces cerevisiae

We chose the parent strain for mutagenesis on the basis of the presence of suitable markers for genetic manipulations and our ability to use extracts prepared from the parental strains for in vitro splicing assays. This feature is important for the subsequent analysis of mutants in the in vitro splicing system. About 1000 temperaturesensitive strains were isolated by ethylmethanesulfonate (EMS) mutagenesis, followed by replica plating on rich medium to screen individual mutagenized yeast colonies for mutants able to grow at $23^{\circ} \mathrm{C}$ but unable to grow at $37^{\circ} \mathrm{C}$. The wild-type parental strain grew at both temperatures. The dose of EMS and time of mutagenesis were selected to give between 10 and $30 \%$ survival after mutagenesis. The temperature-sensitive lethal mutants represented $\sim 1-2 \%$ of the survivors. The mutagenesis was done on a pair of congenic strains of opposite mating types so that half of the temperature-sensitive bank comprised one mating type and the second half comprised the other. Most of the mutants are expected to be of independent origin obtained from nine batches of $\mathrm{mu}$ tagenesis.

\section{Screening of mutants}

We employed a direct assay to screen for mutants that accumulate intron-containing transcripts, not normally seen in a wild-type cell (Fig. 1A). The prp2-prp11 mutants were used as controls for the development of a screen that could reproducibly detect such pre-mRNA splicing mutants. In this way we developed conditions for the temperature shift, RNA preparation, and the detection of intron-containing transcripts. Early-to-midlog phase cells from individual temperature-sensitive strains grown at permissive temperature $\left(23^{\circ} \mathrm{C}\right)$ were shifted to the nonpermissive temperature $\left(37^{\circ} \mathrm{C}\right)$ for $2 \mathrm{hr}$, and harvested. Analysis of the RNAs, present at nonpermissive temperature, was done after a rough estimation of total RNA. Approximately equal amounts of total RNA were fractionated on denaturing agarose gels and subsequently were blotted onto nylon filters. Two effects of the prp2-prp11 mutations on RNA processing are evident: (1) the unique presence of intron-containing precursors after temperature shift, and (2) a decrease in the processed mRNA levels. The levels of mRNA are decreased to $10-50 \%$ of the levels in wild-type cells (data not shown). Therefore, we chose the criterion of accumulation of intron-containing RNAs as an indicator of RNA-processing defects, a feature common to all of the prp2-prp11 complementation groups.

Then these filters were probed for pre-mRNA with ${ }^{32} \mathrm{P}$-labeled actin intron sequences. Actin was chosen for two reasons: (1) It is an abundant mRNA, and (2) its precursor is processed efficiently. Pre-mRNA and the intermediates of RNA processing are not detected easily in wild-type cells (Domdey et al. 1984; this study). Probing the blots with a labeled fragment of the actin intron allows the detection of pre-mRNA, lariat intermediate, and the released intron (Fig. 1B). The detection of in tron-containing RNA species is indicative of a mutant phenotype.

Several positive mutants that accumulate intron-containing RNAs to varying levels were found (Fig. 2). Of the 1000 temperature-sensitive mutants screened, 36 mutants were candidates for defects in pre-mRNA processing and were chosen for rescreening. All candidate strains from the primary screen then were colony-purified and retested for in vivo temperature-sensitive lethality. Subsequently, total RNAs were prepared from these strains, grown at both permissive and nonpermissive temperature for Northern analysis. Most of the newly isolated mutants showed a temperature-dependent accumulation of pre-mRNA (Fig. 2A, lanes $c$ and d, $e$ and $f, i$ and $j)$. However, in some cases, a splicing defect is evident even at the permissive temperature (Fig. 2, lanes $g$ and $h$ ). In these cases there exists a possibility of having generated two independent mutations, one affecting the RNA processing and another leading to the temperature-sensitive lethality. The splicing phenotype in most mutants is consistent with pre-mRNA accumulation, except for distinct phenotypes observed in the following mutants. ts a 107 appeared to accumulate both the pre-mRNA and intron for actin transcripts (see Segregation analysis, below; Fig. 5B). ts a 319 appeared to have a pleitropic effect on pre-mRNA processing at nonpermissive temperature: Both pre-mRNA and an intron-containing transcript larger than pre-mRNA accumulated (Fig. 2A, lane d). Mutants containing RNA of mobility corresponding to that of the released intron were readily apparent even at the primary screen; these 
A
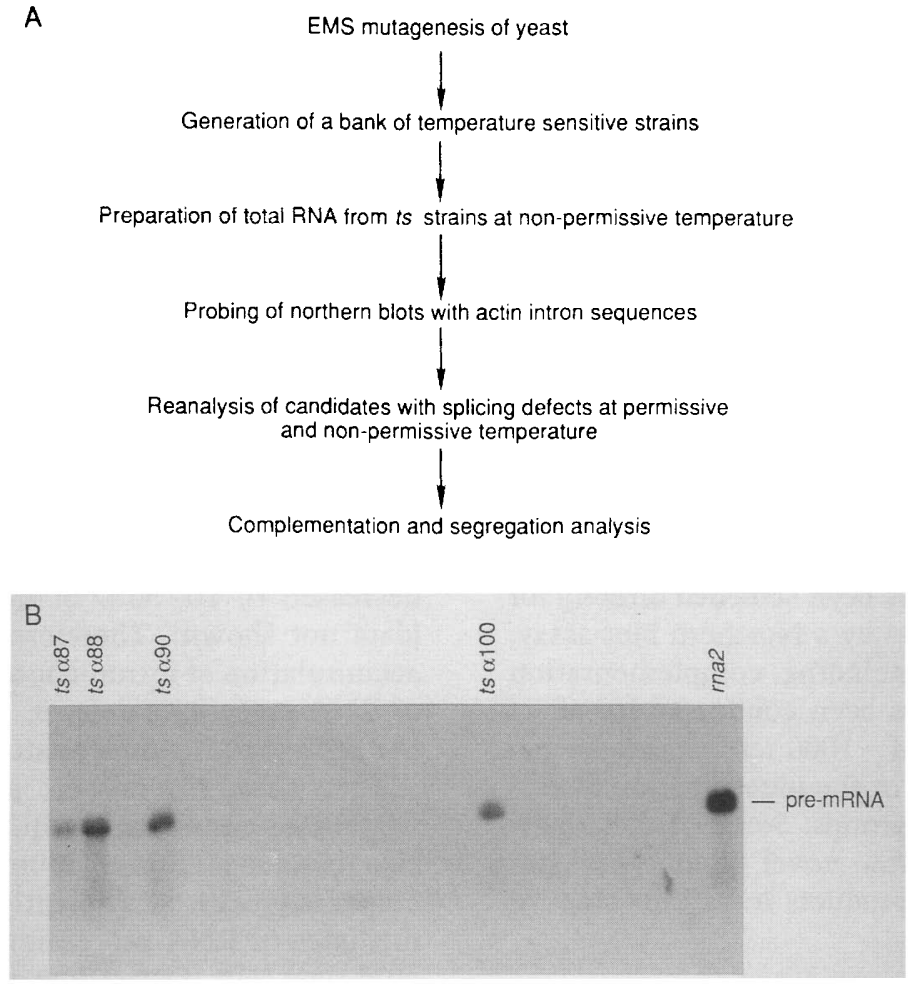

Figure 1. (A) Scheme used for the isolation of splicing mutants; $(B)$ A representative Northern blot from a primary screen of 25 temperature-sensitive mutants. After $2 \mathrm{hr}$ at nonpermissive temperature, $10 \mu \mathrm{g}$ of total RNA from each mutant strain was run on a formaldehyde-agarose gel and then blotted. The probe used was a labeled fragment of the actin intron. The last lane contained total RNA from prp $($ ma $) 2$, a strain known to accumulate pre-mRNAs.

strains did not show any accumulation of pre-mRNA (Fig. 2A, lanes a and b).

Separation of the lariat intermediate from the premRNA for actin transcripts is not sufficiently reproducible in these gels to distinguish between these two species of RNA. Pre-mRNA is $1.7 \mathrm{~kb}$, and the lariat intermediate is $\sim 1.5 \mathrm{~kb}$. Mutants that accumulate lariat intermediates specifically could be identified through primer extension analysis of the RNAs (see below). The levels of mRNA after temperature shift are reduced in

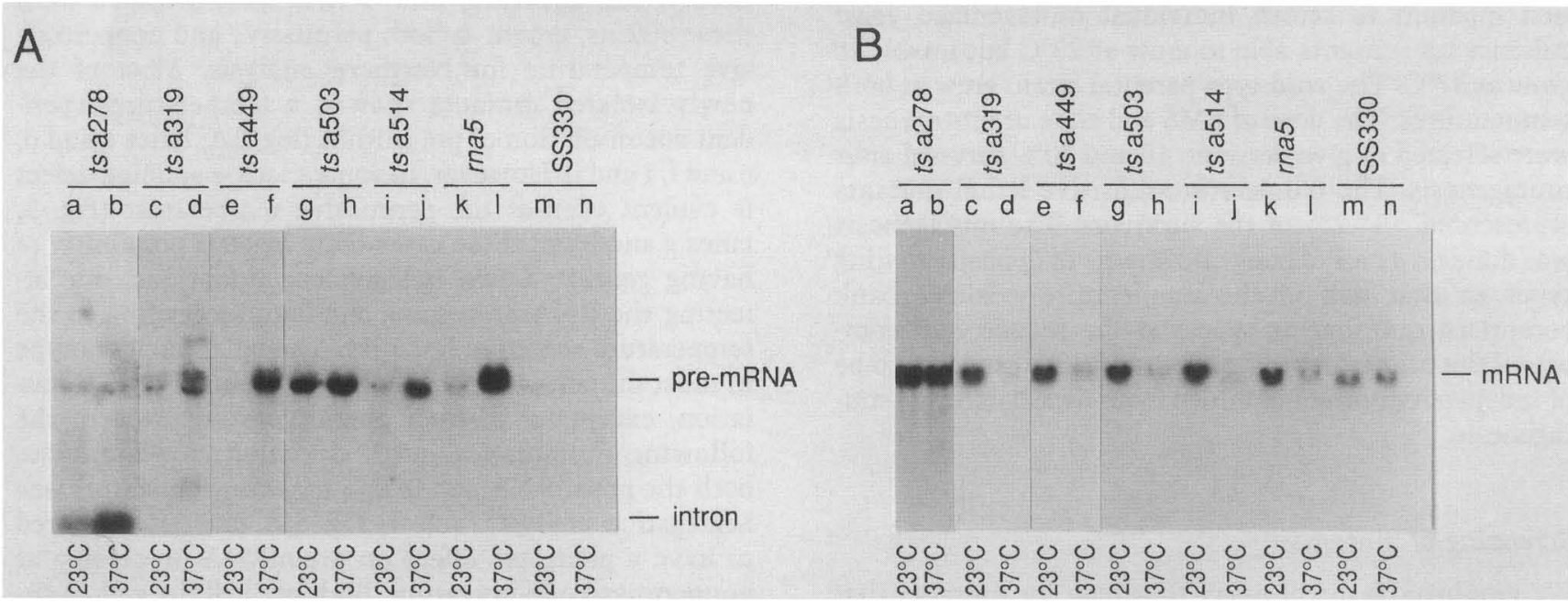

Figure 2. Rescreening, at both permissive and nonpermissive temperatures, of some positive mutants obtained from the primary screen. For each candidate $10 \mu \mathrm{g}$ of total RNA from cells at $23^{\circ} \mathrm{C}$ or after a $2 \mathrm{hr}$ shift to nonpermissive temperature $\left(37^{\circ} \mathrm{C}\right)$ was analyzed. RNAs from wild-type cells and from prp $($ rna $) 5$ at both temperatures also were analyzed as control. $(A)$ The blots were probed with a fragment of the actin intron; $(B)$ a probe for the spliced actin mRNA was used on the same blot as that in $A$. 
all but one of these pip mutants, consistent with the appearance of pre-mRNA (Fig. 2B, lanes c-j). There is not a temperature-dependent change in mRNA levels in the mutant ts a 278, which accumulates intron (Fig. 2B, lanes $a$ and $b$ ).

If the mutations isolated by this screen affect the cellular splicing machinery, intron-containing transcripts other than actin would be expected to accumulate. This was tested by probing Northern blots for a second intron-containing transcript, RP59 (Larkin and Woolford 1983). As expected, an increase in pre-mRNA or the lariat intermediate was observed, together with a dramatic decrease in the level of the mature mRNA at nonpermissive temperature (data not shown). All of these mutants were specific for their effect on pre-mRNA. Non-intron-containing transcripts from the $T C M l$, $P A B l$, and STE2 loci did not accumulate (M. Aebi, pers. comm.).

\section{Piimei extension analysis}

To map precisely the $5^{\prime}$ ends of the accumulated RNAs, primer extension analyses were conducted. These experiments allowed us to distinguish between mutants that specifically accumulate the pre-mRNA and those that accumulate the lariat intermediate. An oligonucleotide primer, complementary to the sequences in the RP51A intron, 70 bases downstream of the $5^{\prime}$ splice site (for RP51A sequence, see Teem and Rosbash 1983), was used for primer extension. Total RNA prepared from cells at both permissive and nonpermissive temperature were used. In wild-type cells a very low level of the extension products corresponding to the pre-mRNA or the lariat intermediate of RP51A was observed (Fig. 3A, lanes a and $\mathrm{b}$ ). In the prp mutants $t s$ a 47 , $t s$ a 107 , ts a 449, ts a 514 , ts a 87, and ts a 344, an increased level of premRNA was seen upon temperature shift (e.g., see Fig. $3 \mathrm{~A}$, lanes $\mathrm{g}$ and $\mathrm{h}$ ). Two primer extension products corresponding to pre-mRNA were obtained, representing RNAs initiated from the two transcription start sites of RP5IA (Teem and Rosbash 1983). An increased level of the lariat intermediate was seen at permissive temperature in some of the strains that accumulate pre-mRNA at nonpermissive temperature. This could be indicative of low splicing efficiency, even at the permissive temperature.

In the strains $t s$ a 452, ts a 503, ts a 365, ts a 491, and ts a 487, only a single extension product corresponding to the $5^{\prime}$ end of the RP5IA intron was detected (e.g., see ts a 503, in Fig. 3A, lanes e and f). These strains had exhibited accumulation of a large, intron-containing actin transcript (-1.5 kb; Fig. 2A, lanes g and h) on
A
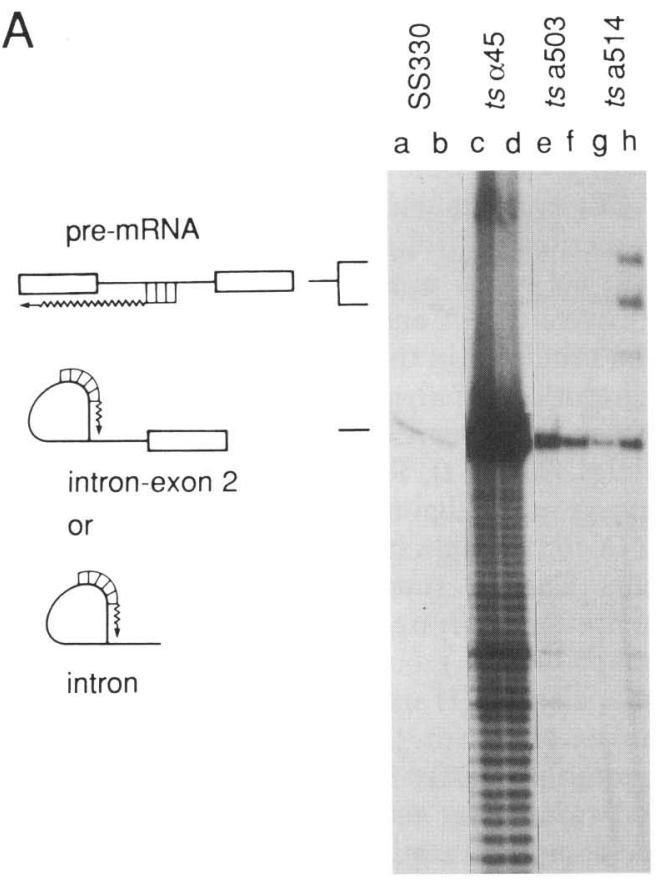

OO OOOONO
B
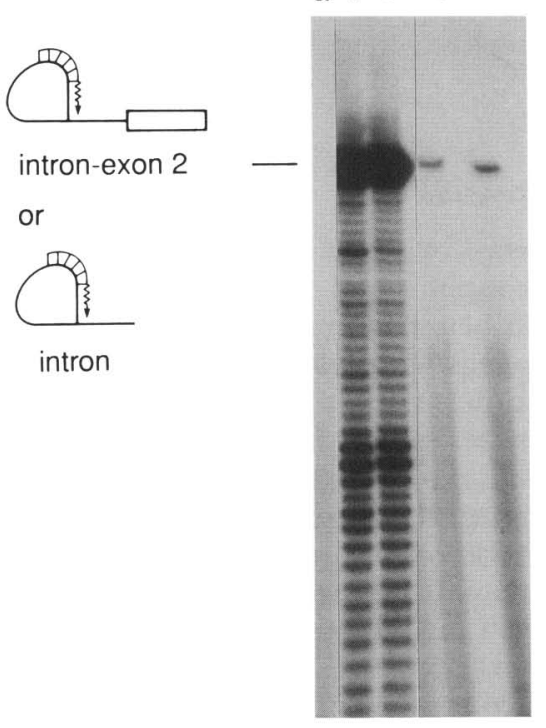

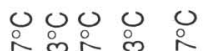

Figure 3. [A Reverse transcription using a primer, 5' of the RP51A branch point. Total RNA (20 $\mathrm{xg}$ ) prepared from each of the four different strains denoted above the lanes (SS330, ts a 45, ts a 503, ts a 5147 was used for primer extension. RNA from cells at both permissive and nonpermissive temperature was analyzed, as indicated at bottom. The primer extension stops corresponding to 5 ' ends of lariat molecules or $5^{\prime}$ ends of the pre-mRNA are shown by schematic representations. $(B)$ Reverse transcription from a primer, 5 ' of the actin branchpoint. Total RNAs (20 jxg) from the wild-type SS330 strain, the mutant ts a 391 accumulating the released intron, and the strain $t s$ a 503 that accumulates the lariat intermediates were used. A schematic representation of the primer extension stop is shown. 
Northern blots. To verify that this $\sim 1.5-\mathrm{kb}$ actin RNA represented the lariat intermediate of actin, further primer extension experiments were performed with an oligonucleotide primer complementary to the actin intron sequences 70 nucleotides downstream of the $5^{\prime}$ splice site (for actin sequence, see $\mathrm{Ng}$ and Abelson 1980). A dideoxy sequencing experiment, using the same primer, on an actin M13 clone allowed a precise mapping of the $5^{\prime}$ splice site. These experiments confirm the accumulation of the lariat intermediate of the actin transcript (Fig. 3B, lanes $\mathrm{d}$ and e). The primer extension experiment with RP51A transcripts and actin transcripts indicated that there was no increase in the level of fulllength precursor in these mutants. This suggests that, in these mutants, the first step of splicing: Cleavage at the 5 ' splice site proceeds normally, but the second step of splicing is defective. This phenotype is evident at both permissive and nonpermissive temperature and does not affect the precision of cleavage at the $5^{\prime}$ splice site. These results also confirm that the primary defect in these strains is accumulation of the lariat intermediate of splicing with no effect on the levels of the pre-mRNA.

The mutants $t s$ a 278, ts a 391, ts $\alpha 45$, and $t s \alpha 397$, accumulated the released intron specifically (Fig. 2A, lanes $\mathrm{a}$ and $\mathrm{b}$ ). Primer extensions again demonstrated a $5^{\prime}$ terminus in these accumulated RNAs corresponding to the $5^{\prime}$ end of the intron (e.g., see Fig. 3A, lanes $\mathrm{c}$ and d; Fig. 3B, lanes $b$ and $c$ ). The accumulation of the intron was observed at both permissive and nonpermissive temperature. Reverse transcription with an oligonucleotide that hybridized $3^{\prime}$ to the branch consensus of RP51A or actin indicates that these molecules are branched at the correct site and therefore do not represent imprecisely spliced or branched molecules (data not shown).

\section{Complementation analyses}

The mutants picked by this screen were backcrossed to the appropriate congenic wild-type strain of the opposite mating type, and heterozygous diploids were obtained. The putative diploids were tested for diploidization and for the temperature-sensitive phenotype. All diploids were temperature resistant, implying that the temperature-sensitive mutation is recessive in all of these strains. No dominant temperature-sensitive mutants were isolated in this screen. To define which of the newly isolated mutants belonged to known complementation groups, each mutant strain was crossed to prp2prp11 mutants of the opposite mating type, and the diploids were isolated on the appropriate selective media. The diploids subsequently were screened at permissive and nonpermissive temperature. Noncomplementing diploids exhibit a temperature-sensitive phenotype and thus show that the pair of recessive mutants belong to the same complementation group (Fig. 4). The complementation analysis showed that six mutants were prp2, seven were $p r p 3$, six were $p r p 6$, and two were $p r p 9$. No new isolates of prp4, prp5, prp7, prp8, or prp11 were obtained (Table 1). Mutants that complemented the prp2-

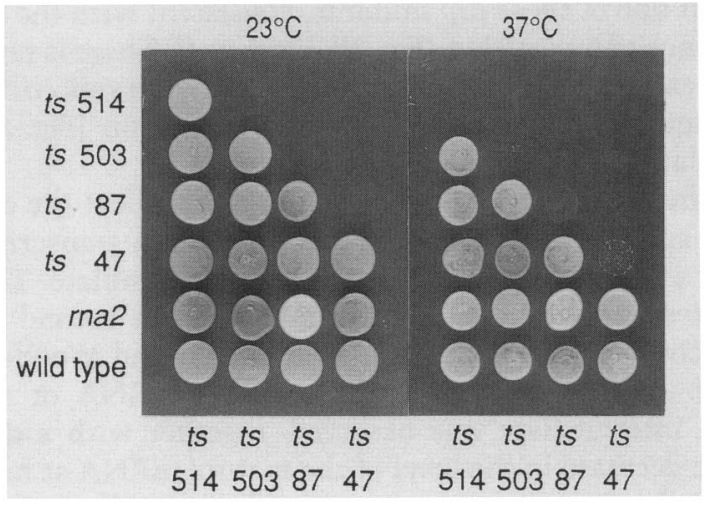

Figure 4. Complementation analysis of prp mutants. Diploids generated from all pairwise crosses of $t s 47, t s 87, t s 503, t s 514$, prp (rna) 2 and the wild-type strain. (left) Growth at permissive temperature $\left(23^{\circ} \mathrm{C}\right)$; (right) growth of the same dioploids at nonpermissive temperature $\left(37^{\circ} \mathrm{C}\right)$.

prp11 (all pairs of diploids are $\mathrm{TS}^{+}$) were taken to represent new complementation groups (see Table 1). ts a 100 , a mutant that accumulates pre-mRNA, had a mating defect and, hence, has not been assigned to any group. The new mutants then were assigned to complementation groups. First, the MATa and MAT $\alpha$ mutants were crossed. We determined that the mutants $t s$ a 452 , ts a 503, and ts $\alpha 491$ belong to the same complementation group. For the remaining mutants, which were in one mating type, corresponding mutants of the opposite mating type were constructed. Then we crossed the $M A T$ a mutants with $M A T \alpha$ mutants and assigned complementation groups. All of these complementation results are based on the assumption that the temperaturesensitive defect and the splicing phenotype are caused by a mutation in the same gene in these mutants. In our segregation analysis (below), two complementation groups were represented by non-temperature-sensitive alleles (Table 1). Reaffirmation of their complementation group assignment was made after analysis of the RNA for the splicing phenotype in the appropriate diploids. Table 1 summarizes the results of the complementation analysis. We found 11 new complementation groups. These groups can be divided into four phenotypic classes: (1) six complementation groups that accumulate precursor; (2) two complementation groups that accumulate lariat intermediate; (3) two complementation groups that accumulate the released intron; and (4) one complementation group that accumulates both premRNA and intron.

\section{Segregation analysis}

Mutants that complement prp2-prp11 were analyzed further to determine whether single-site mutations were responsible for both the splicing phenotype and temperature sensitivity. The diploids that were heterozygous for a prp mutant were sporulated. Tetrad analysis 
Table 1.

\begin{tabular}{|c|c|c|c|}
\hline Complementation group & Number of alleles & Splicing defect & Transcripts tested \\
\hline $\operatorname{prp}(r n a) 2$ & 6 & pre-mRNA & actin \\
\hline $\operatorname{prp}(r n a) 3$ & 7 & pre-mRNA & actin \\
\hline prp(rna)6 & 6 & pre-mRNA & actin \\
\hline $\operatorname{prp}(\mathrm{ma}) 9$ & 2 & pre-mRNA & actin \\
\hline prp(rna) $17($ ts 365, ts 487$)$ & 2 & lariat-intermediate & actin, RP51A \\
\hline $\operatorname{prp}(r n a) 18$ (ts 452, ts 503, ts 491) & 3 & lariat-intermediate & actin, RP51A, RP59 \\
\hline prp(rna)19 (ts 87) & 1 & pre-mRNA & actin, RP51A \\
\hline $\operatorname{prp}(r n a) 20$ (ts 319) & 1 & pre-mRNA and transcript larger than pre-mRNA & actin, RP59 \\
\hline $\operatorname{prp}(r n a) 21$ (ts 47) & 1 & pre-mRNA & actin, RP51A, RP59 \\
\hline prp(rna)22 (ts 107) & 1 & pre-mRNA and intron & actin, RP51A, RP59 \\
\hline prp(rna)23 (ts 514) & 1 & pre-mRNA & actin, RP51A, RP59 \\
\hline prp(rna) 24 (ts 344) & 1 & pre-mRNA & actin, RP51A \\
\hline$p r p(m a) 25$ (ts 449) & $1^{2}$ & pre-mRNA & actin, RP51A, RP59 \\
\hline prp (rna)26 (ts 278, ts 391, ts 45$)$ & $3^{a}$ & intron & actin, RP51A, CYH2 \\
\hline $\operatorname{prp}($ rna $) 27$ (ts 397) & 1 & intron & actin, RP51A \\
\hline
\end{tabular}

${ }^{a}$ None of the alleles is temperature sensitive.

showed that the temperature-sensitive defect segregated $2: 2$ in all cases $(10-20$ tetrads analyzed in each case), as did all other heterozygous markers, indicating a defect in a single nuclear gene. Cosegregation of the splicing phenotype with the temperature-sensitive defect also was analyzed for all the new complementation groups. RNAs from several tetrads (in each case, six to nine tetrads) and from the heterozygous diploid were analyzed after temperature shift on Northern gels (Fig. 5A-D). Appearance of the splicing defect in the temperaturesensitive spores is indicative of cosegregation of the two phenotypes. The heterozygous diploids did not display a gene dosage effect for either the temperature-sensitive or the prp phenotype (data not shown). Cosegregation was observed for the precursor-accumulating strains ts a 47 (Fig. 5A), ts a 107 (Fig. 5B), ts a 319, ts a 514, ts $\alpha$ 87, and ts $\alpha 344$. The lariat intermediate-accumulating mutants ts a 503 (Fig. $5 \mathrm{C}$ ) and $t s \alpha 365$, representing the two complementation groups, also displayed cosegregation of the splicing and temperature-sensitive phenotype.

Among the intron-accumulating mutants, only one $t s$ a 397 (Fig. 5D), exhibited cosegregation of the splicing and temperature-sensitive phenotypes. The accumulation of intron in the strains ts a 278, ts a 391, and ts $\alpha 45$ was caused by a single nuclear recessive gene but was not linked to the temperature-sensitive defect. This result implies that the four isolates of the intron-accumulating mutant possibly represent one complementation group with temperature-sensitive and non-temperaturesensitive alleles. Diploids obtained from crosses of each of the intron mutants to all of the others and also to all of the splicing mutants were analyzed for the presence of the intron phenotype at nonpermissive temperature. Intron accumulation phenotype was observed only in diploids obtained from pairwise crosses using $t s$ a 278, ts a 391 , and $t s \alpha 45$. These results indicated that the intron mutants ts a 278, ts a 391, and ts $\alpha 45$ represent one new complementation group and the mutant $t s \alpha 397$ represents another (Table 1).

The precursor accumulation in the mutants ts a 449 and $t s \alpha 377$ was not linked to the temperature-sensitive lethality, and the isolation of these mutants as temperature-sensitive was serendipitous. An analysis of the RNA in diploids similar to that done for the intron mutants was conducted. These results indicated that the mutants ts a 449 represented a new complementation group and is not a non-temperature-sensitive allele of another complementation group. On the other hand, $t s \alpha$ 377 proved to be a non-temperature-sentsitive allele of prp2, a previously known complementation group.

\section{Discussion}

The enumeration, identification, and determination of function of the splicing components is essential for the complete understanding of the pre-mRNA splicing process. The complexity of the spliceosome is revealed by biochemical work indicating the involvement of five snRNPs (U1, U2, U4, U5, and U6), heterogeneous nuclear ribonucleoproteins (hnRNPs), and several protein factors (Green 1986; Maniatis and Reed 1987; Sharp 1987). The extensive repertoire of genetic techniques applicable to the yeast $S$. cerevisiae allows the combined approach of biochemistry and genetics in analysis of premRNA splicing in yeast. Analysis of the yeast mutants in 10 complementation groups (prp2-prp11) over several years has made it clear that primary defect in these strains is the accumulation of intron-containing premRNAs at nonpermissive temperature (Rosbash et al. 1981; Teem et al. 1983; Miller 1984). Evidence for the direct participation of many of these gene products in mRNA splicing comes from demonstrating that extracts from seven of these mutant groups can be specifically heat-inactivated in vitro (Lustig et al. 1986).

Our direct screening of a temperature-sensitive bank of yeast strains for splicing mutants has resulted in the isolation of four classes of mutants affecting different stages of the splicing reaction pathway. The major class of six new complementation groups share the phenotype 


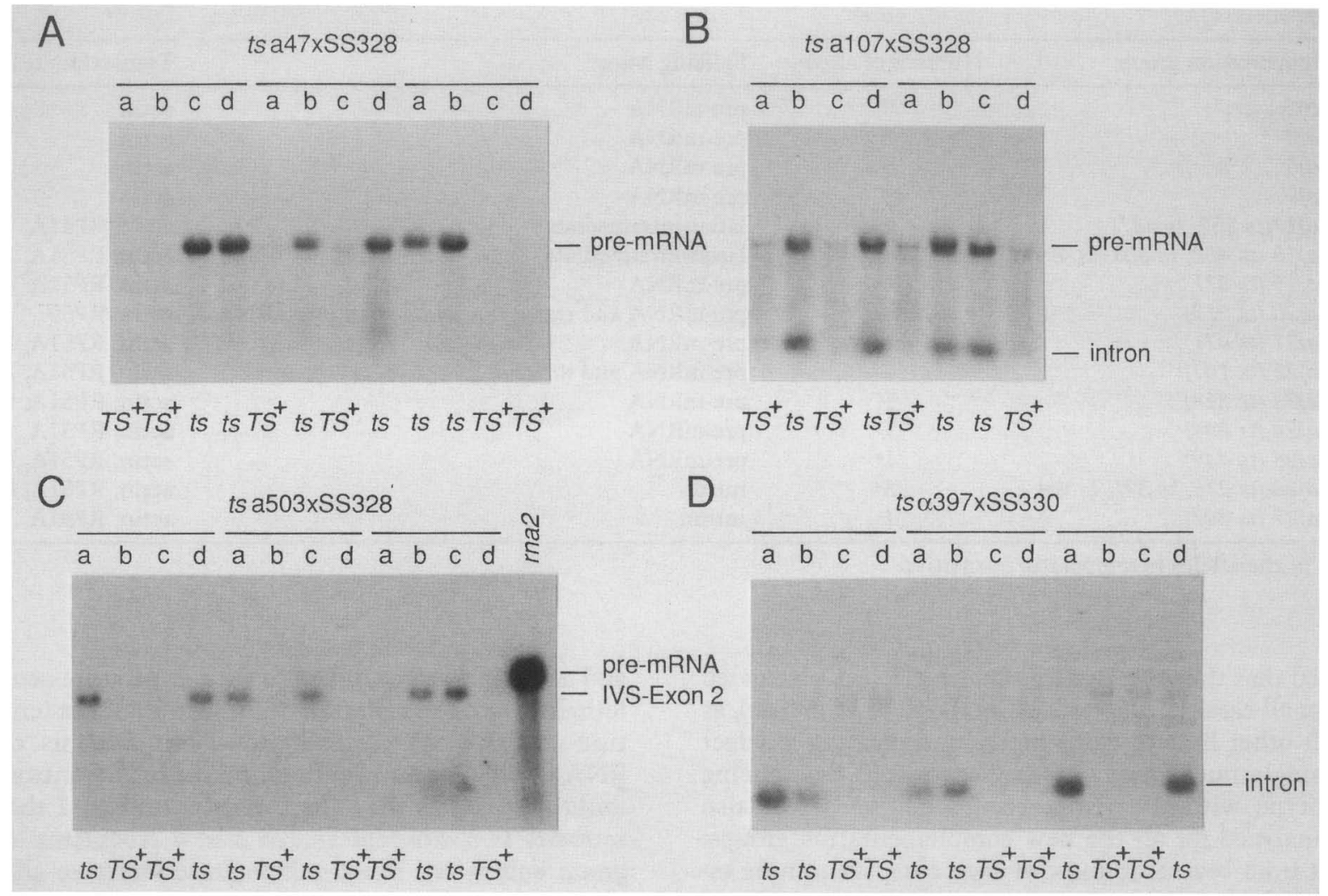

Figure 5. Cosegregation of the temperature-sensitive and splicing phenotypes. An example from each of the four phenotypic classes of splicing mutants is shown. Heterozygous diploids were sporulated, and tetrads dissected in each case. Spores from several tetrads were grown to log phase and shifted to nonpermissive temperature for $2 \mathrm{hr}$ before RNA extraction. The RNAs were assayed on Northern blots for the splicing phenotype. $(A)$ Pre-mRNA accumulation; $(B)$ pre-mRNA and intron accumulation; $(C)$ lariat intermediate accumulation (IVS-Exon2); $(D)$ intron accumulation. The growth phenotype of the spores at $37^{\circ} \mathrm{C}$ is indicated also.

of the prp2-prp11 groups (pre-mRNA accumulation). Two novel classes affecting late events in splicing were obtained. One class accumulates the lariat intermediate of splicing, and the other accumulates the released lariat intron, with no significant increase in pre-mRNA. One mutant was obtained that could be classified as both a pre-mRNA and an intron-accumulating strain.

\section{New temperature-sensitive mutations define genes involved in various stages of pre-mRNA splicing}

The prp2-prp11 mutants were identified as being defective in RNA metabolism, and their isolation was not due to a specific screen for mutants in pre-mRNA splicing (for review, see Warner 1987; Vijayraghavan and Abelson 1989). Of the initial collection of temperature-sensitive lethal mutants generated by nitrosoguanidine mutagenesis, $\sim 5 \%$ were defective in splicing (Hartwell et al. 1970). We screened our temperature-sensitive bank specifically for identification of mutants in pre-mRNA splicing. The screening of the temperature-sensitive mutants for the presence of intron-containing transcripts was a rational approach because all of the previously identified complementation groups (prp2prp11), shared the phenotype of pre-mRNA accumula- tion at nonpermissive temperature. We isolated 36 mutants defective in pre-mRNA splicing that represented both old and new complementation groups.

\section{Temperature-sensitive mutations that cause pre-mRNA accumulation}

The majority of the new complementation groups shared the same phenotype of pre-mRNA accumulation as seen in the prp2-prp11 mutants. The large number of mutants obtained that affect early steps in the splicing pathway could be due to the requirement for several factors early in spliceosome assembly. These may range from components that are direct participants in the splicing machinery, to components that perform essential modifications of the spliceosomal components, or to components of hnRNP complexes and factors involved in only the assembly of the spliceosome and not in later functions of the spliceosomes. An alternative reason could be the presence of shared components between the early and late steps of the splicing pathway. In spite of these reasons, the high proportion of the temperaturesensitive mutations that leads to splicing defects needs some explanation. It may be that some of these mutants affect splicing indirectly; however, of the original 10 
complementation groups, at least 7 appear to affect splicing directly (Lustig et al. 1986). The multicomponent nature of the spliceosome and the need for its assembly may require a large number of genes. These genes may have an unusually large number of sites that can give rise to temperature-sensitive lethal mutations; other genes may have fewer sites that give rise to temperature-sensitive mutations. We prefer to believe that much less than $5 \%$ of the genes in S. cerevisiae are required for mRNA splicing.

Some of the mutants that we isolated by this screening procedure are not temperature sensitive. From our temperature-sensitive bank, we isolated one new complementation group that accumulates pre-mRNA but results in this splicing defect without a severe growth defect at $37^{\circ} \mathrm{C}$. In addition, we obtained mutants (discussed below) that accumulate intron, but are not temperature sensitive, and also a non-temperature-sensitive allele of a previously identified mutant, prp2. It has been shown that pre-mRNA splicing is not the ratelimiting step in mRNA synthesis (Pikielny and Rosbash 1985). Some defects in pre-mRNA splicing are apparently not severe enough to limit growth seriously.

Not all of the prp2-prp11 complementation groups were represented in this screen of EMS induced temperature-sensitive mutations. However, a similarity exists in the distribution of allele numbers in prp2, prp3, and prp9 groups between this and the previous study (Harwell et al. 1970). In both cases, a significantly higher number of prp2 and prp3 alleles were obtained than alleles in other complementation groups. One difference is the higher frequency of isolation of prp6 in this study. prp7, prp8, and prp11 were represented by single isolates in the Hartwell screen, and no alleles of these mutants were picked up in this screen. By employing a direct screen, we obtained new complementation groups that affect late events of splicing. Four complementation groups exhibit the novel phenotype of lariat intermediate or intron accumulation. The Hartwell screen employed for the isolation of the prp2-prp11 mutants was based on the ratio of protein/RNA incorporation being $>4$; therefore, it is not surprising that mutants accumulating intron were not in that collection. The levels of mRNA and, hence, protein synthesis in the intron-accumulating mutants are probably unaffected within $2 \mathrm{hr}$ of temperature shift. The isolation of these new complementation groups in our search for splicing mutants might be a result of both the direct screen employed and the mutagen used.

\section{Temperature-sensitive mutations that cause lariat intermediate accumulation}

The isolation of mutants with a novel phenotype affecting only the second reaction of splicing allows an opportunity for the investigation of factors required specifically after the spliceosome assembly and the first step of splicing. Two complementation groups with this phenotype were obtained. All mutants in these groups are temperature sensitive. The precision of the $5^{\prime}$ splice site and the branchpoint selection was not affected, and use of the wild-type $5^{\prime}$ splice site and branch formation site was observed. No change in the levels of the pre-mRNA occurred; hence, the effects seemed to be specific for the second step of splicing, and the presence of the lariat intermediate in splicing was probably not a result of slower splicing. The alleles were partly defective at the permissive temperature and showed increased levels of the lariat intermediate. The second step of splicing leading to formation of mRNA and lariat intron takes place, although inefficiently, at the permissive temperature. A substantial decrease in the second splicing reaction and, hence, the mRNA levels takes place upon temperature shift.

These are the only trans-acting mutants known to act in the splicing pathway after the $5^{\prime}$ splice site cleavage. From the previous studies on the analysis of the spliceosome assembly, one would expect heat-treated extracts from these mutants to accumulate the active spliceosome containing exon 1 and the lariat intermediate $(\mathrm{Pi}$ kielny et al. 1986; Cheng and Abelson 1987). None of the prp2-prp11 mutants undergoes any of the splicing reactions, and most are required early in spliceosome assembly (Lin et al. 1987). Of the prp2-prp11 mutants, the $P R P 2$ gene product is the only one known to be required after the formation of a late intermediate of spliceosome assembly (Lin et al. 1987). Heat-inactivation experiments with prp2 had demonstrated the accumulation of spliceosomes containing the pre-mRNA with both steps of splicing blocked. The newly defined complementation groups that accumulate the lariat intermediate must represent gene products that act after $P R P 2$ in the spliceosome assembly pathway. Chasing experiments with heat-inactivated prp2 spliceosomes have led to the proposal that two distinct sets of factors are required for the second step of splicing reactions (Lin et al. 1987). One of these factors, fraction III, had been identified earlier through fractionation of wild-type extracts /Cheng and Abelson 1986). The second set of factors, termed $C_{n}$ comprise an unidentified set of factors. The temperature-sensitive mutations in two complementation groups accumulating the lariat intermediate possibly result from mutations in one of these factors predicted to act late in the splicing pathway.

\section{Stabilization of the released intron}

Apart from the isolation of mutants required for the second step of splicing, new complementation groups were obtained, with the novel phenotype identifying gene products required after splicing has been completed. The phenotype of intron accumulation was observed in two complementation groups. One group is represented by a single allele and is temperature sensitive, whereas the other consists of three alleles, none of which is temperature sensitive (Table 1). Wild-type cells appear to degrade the intron very rapidly, with no detectable amount of the released intron in the cells. The accumulation of the intron implies that it must be stabilized. The intron-accumulating phenotype does not re- 
sult from the accumulation of aberrantly formed lariats due to the imprecise use of the $5^{\prime}$ splice site or the branch acceptor nucleotide (Fig. 3). The stabilization of the intron could result from its association with spliceosomal components due to a defect in the disassembly or from a defect in the debranching function itself. Analysis of the biochemical phenotype should lead to a better understanding of the nature of these mutations. An additional mutant (ts a 107) shows accumulation of both pre-mRNA and some intron. It probably results from a mutation in a factor required early in splicing. The accumulation of intron in this mutant may result from the continued association of this factor with released intron. Altenatively, this mutation may somehow affect the recycling of spliceosomal factors after the release of the intron.

\section{Isolation of new alleles of prp2, prp3, prp6, and prp9}

The 21 alleles corresponding to the previously identified complementation groups (prp2-prp11) were identified solely on the basis of complementation of the temperature-sensitive or prp phenotype in the diploid. In multicomponent systems, like pre-mRNA splicing, the possibility that the temperature-sensitive or the prp phenotype in the diploid is due to unlinked noncomplementation needs to be considered (for other examples in yeast, see Atkinson 1985; Stearns and Botstein 1988). Thus, it is possible that some of the mutants now thought to be in the prp2-prp11 complementation groups could actually represent new mutations in components interacting with the PRP2-PRP11 gene products. This question can easily be addressed genetically through the recombination analysis of spores from temperature-sensitive diploids. The isolation of any noncomplementing unlinked mutants would be extremely interesting, as these mutants might define components that interact with $P R P 2, P R P 3, P R P 6$, and $P R P 9$ gene products. These mutations resulting in pre-mRNA accumulation could define components that are themselves a part of the splicing machinery.

The assignment of $t s \alpha 100$ and ts $\alpha 200$ to the prp9 complementation group is tentative, because it is uncertain that the original prp9-1 mutation was due to a single-site temperature-sensitive mutation (Hartwell et al. 1970). Confirmation of the assignment of the putative $\operatorname{prp} 9$ alleles should be done easily once the genetic analysis of the original prp9-1 allele is complete.

In conclusion, we isolated more splicing mutants affecting various stages of the splicing pathway. Of these, several complementation groups (prp17, prp18, prp26, and prp27) specifically affect splicing after spliceosome assembly. The isolation of multiple alleles in some of these groups suggests that few components are required specifically for the later stages of splicing. Two avenues of research are now open to further characterize the new mutants. The cosegregation of the prp and the temperature-sensitive phenotype provides a good opportunity to study the function of these gene products through molecular cloning. In parallel, the in vitro characterization of the mutants would also be useful in giving a more detailed understanding of these phenotypes. Both of these complementary approaches are achieved easily in yeast with the existence of in vitro systems and the extensive molecular genetic techniques.

\section{Materials and methods}

Yeast strains

Table 2 lists the strains used in this study. The source of these strains is described in Lustig et al. (1986).

\section{Genetic manipulations}

The media, rich YPD, omission, presporulation, and sporulation were prepared as described in Sherman et al. (1986). Complementation analysis was carried out by mixing pairs of prp mutants of opposite mating type on YPD plates and allowing mating and diploid growth at $23^{\circ} \mathrm{C}$ overnight. The diploids were obtained from minimal plates supplemented for their selection and were then tested for growth at $37^{\circ} \mathrm{C}$ on YPD plates. In cases where necessary, complementation of the prp phenotype in the diploids was assessed by Northern analysis. Diploids for the analysis of dominant versus recessive nature were obtained at permissive temperature from selective media and then tested for their temperature sensitivity. Sporulation of the heterozygous diploids was done at $23^{\circ} \mathrm{C}$, and dissection of spores was done by standard procedures also at $23^{\circ} \mathrm{C}$.

\section{Mutagenesis}

The two strains, wild type for growth at $37^{\circ} \mathrm{C}$, SS330 and SS328, were mutagenized, with the alkylating agent EMS, essentially as in Moir et al. (1982). Stationary-phase cells were resuspended at $10^{8} \mathrm{cells} / \mathrm{ml}$ in $0.1 \mathrm{M}$ sodium phosphate buffer $(\mathrm{pH} 7.0)$ and treated with $3 \%$ EMS for $\sim 60 \mathrm{~min}$. This treatment was calibrated to give $10-30 \%$ survival for these strains after the procedure. Mutagenized cells were first diluted 40 -fold in sterile $5 \%$ sodium thiosulfate to inactivate the mutagen. This was followed by dilutions in sterile water and plating for growth on YPD plates at $23^{\circ} \mathrm{C}$. Plates with $\sim 200$ colonies were replica plated on prewarmed YPD plates and left overnight at $37^{\circ} \mathrm{C}$. Colonies that grew poorly or did not grow at $37^{\circ} \mathrm{C}$ were retested for the growth defect, and the strains that still exhibited poor or no growth at $37^{\circ} \mathrm{C}$ were included in the temperature-sensitive strain collection.

\section{Temperature shift and preparation of RNA}

Each of the temperature-sensitive mutants was grown in a 2-ml YPD culture to early-to-mid-log phase of growth $\left(\mathrm{A}_{600} 0.5-2.0\right)$ and then shifted to nonpermissive temperature $37^{\circ} \mathrm{C}$ for $2 \mathrm{hr}$. Cells were pelleted and then frozen in dry ice. As a control for the temperature-shift experiment, either a prp2 or a prp 5 strain was used. Extraction of the total RNA from the cell pellet was done by the method of hot phenol extraction similar to that of Domdey et al. (1984). Cell pellets were resuspended in $50 \mathrm{mM}$ sodium acetate (pH 5.3) and $10 \mathrm{mM}$ EDTA, vortexed, and SDS was added to $1 \%$. The cell suspension was vortexed and extracted with phenol (equilibrated with $50 \mathrm{~mm}$ sodium acetate and $10 \mathrm{~mm}$ EDTA) at $65^{\circ} \mathrm{C}$ for $5 \mathrm{~min}$. Following centrifugation, the aqueous phase was transferred and extracted with equal 
Table 2. List of yeast strains

\begin{tabular}{|c|c|}
\hline Strain & Genotype \\
\hline SS328 & 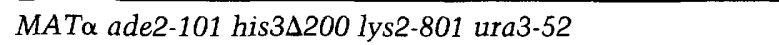 \\
\hline SS330 & MATa ade2-101 his $3 \Delta 200$ tyr1 ura3-52 \\
\hline mal-1 & MATaprp(rna)1-1 adel ade2 his7 tyr1 ura1 \\
\hline RL 92 & MATaprp(ma)2 leu2-3 leu2-112 ura3-52 \\
\hline SPJ 3.33 & MATaprp(rna)3 his3 leu2 lys2 ura3-52 \\
\hline RL144 & MATaprp(rna)4 his3s-1 leu2-3 leu2-112 lys2 tyr1 uras 3-52 \\
\hline RL173 & MATaprp(rna)5 ade his7 leu2 lys2 \\
\hline ma 6-1 & MATaprp(rna)6-1 ade1 ade2 his7 lys2 tyr1 ura1 \\
\hline ma $7-1$ & MATaprp(rna)7-1 ade1 ade2 his7 lys2 tyr1 ura1 \\
\hline ma 8-1 & MATaprp(rna)8-1 ade1 ade2 his 7 lys2 tyr1 ura1 \\
\hline ma 9-1 & MATaprp(rna)9-1 ade1 ade2 his7 lys2 tyr1 ura1 \\
\hline SPI 11.4 & MATaprp(rna)11 his4-512 leu2 ura3-52 \\
\hline ts $136 \# 7 \mathrm{~A}$ & MATaprp(rna) 1 ade his met trp ura \\
\hline SS304 & MAT $\alpha$ prp(rna)2 ade2-1 his3-532 trp1-289 ura3-1 ura3-2 \\
\hline $\operatorname{ma} 3-3 \mathrm{~A}$ & MAT $\alpha$ prp(rna)3 ade leu \\
\hline SPJ 4.43 & MATaprp(rna)4 ade1 ade2 his7 leu2 lys2 ura3-52 \\
\hline SPJ 5.41 & MATaprp(rna)5 his3 his7 leu2 ura3-52 \\
\hline SPJ 6.68 & MATaprp(rna)6 his3 lys2 ura3-52 \\
\hline $\operatorname{ma} 7(\mathrm{AH})$ & MAT $\alpha p r p(r n a) 7$ ade arg leu ura1 \\
\hline $\operatorname{ma} 8(\mathrm{AH})$ & MAT $\alpha p r p(r n a) 8$ ade arg trp ura1 \\
\hline SPJ 10.2 & MATaprp(rna)11 ade his leu lys tyr ura \\
\hline
\end{tabular}

volumes of phenol/chloroform (1:1), at room temperature for 5 min. After centrifugation, the aqueous phase was brought to 0.3 M sodium acetate and the RNA was precipitated with 2.5-3 volumes of ethanol. The RNA yield was usually $50-200 \mu \mathrm{g}$ from this small-scale preparation. Rescreening of the positive mutants from the first screen was done at both temperatures. Here, an aliquot of the mid-log phase cells was shifted to nonpermissive temperature for $2 \mathrm{hr}$ and the rest of the culture maintained at permissive temperature. Extraction of RNA was done as described above from both cultures.

\section{Northern analysis}

Approximately $10 \mu \mathrm{g}$ of total RNA from each mutant at nonpermissive temperature was used for Northern analysis on formaldehyde-agarose gels. The RNA was added to formamide dye $\mathrm{mix} 150 \%$ formamide, $6 \%$ formaldehyde, $50 \mathrm{~mm}$ HEPES at $\mathrm{pH} 7.4,1 \mathrm{~mm}$ EDTA, $20 \%$ glycerol, and $0.1 \%$ of the dyes bromophenol blue and xylene cyanol) and heated at $65^{\circ} \mathrm{C}$ for 2 min before loading on gels. The electrophoresis was done in $1.2 \%$ agarose gels in $50 \mathrm{mM}$ HEPES, $1 \mathrm{mM}$ EDTA, and $6 \%$ formaldehyde. Following electrophoresis, the RNA was blotted onto GeneScreen membranes in $25 \mathrm{~mm}$ sodium phosphate $(\mathrm{pH} 6.5)$. The RNA was cross-linked on the membranes, as described in Cheng and Abelson (1987).

Prehybridization, hybridization, and washing of the filters was done as described in Cheng and Abelson (1987). The blots were probed with a $\sim 200$-bp fragment of the actin intron (XhoI-ClaI of actin intron; for actin sequence, see $\mathrm{Ng}$ and Abelson 1980l, labeled by the random primer extension method of Feinberg and Vogelstein (1983) and used for the detection of the pre-mRNA, lariat intermediate, and the intron. The detection of the mRNA and the pre-mRNA was done with a labeled fragment of the actin clone. Reprobing of the blots was done after removal of the probe by boiling in $0.1 \times$ SSC, $0.1 \%$ SDS for 5-10 min.

\section{Reverse transcription}

Oligonucleotides used for the primer extension were as given below: RP51A: 5'-GTATGACTTTATTGCGCATGTCGACTC-
3'; actin XhoI: 5'-CTCTCGAGCAATTGGGACCGTGC-3'. The primer oligonucleotides $(20 \mathrm{ng})$ were $5^{\prime}$-end-labeled using [ $\left.\gamma^{-32} \mathrm{P}\right]$ ATP (crude $7000 \mathrm{Ci} / \mathrm{mm}$ ) and T4 polynucleotide kinase. The labeled oligonucleotides were purified on DE-52 ion exchange column and ethanol-precipitated. Total RNA $(20-25 \mu \mathrm{g}$ was used in each primer extension with $\sim 50,000 \mathrm{cpm}$ of the primer in $50 \mathrm{~mm}$ Tris- $\mathrm{HCl}(\mathrm{pH} 8), 150 \mathrm{mM} \mathrm{KCl}, 0.5 \mathrm{~mm}$ EDTA, $1 \mathrm{~mm} \mathrm{DTT}$, and $7 \mathrm{~mm} \mathrm{MgCl}$. Annealing was done by heating the RNA, primer, and buffer at $90^{\circ} \mathrm{C}$ for $2 \mathrm{~min}$, followed by slow-cooling to room temperature. Fifteen to twenty units of AMV reverse transcriptase and $0.5 \mathrm{~mm}$ of each dNTP were added, and the extensions done at $42^{\circ} \mathrm{C}$ for $45 \mathrm{~min}$. The extension products were separated on 8 or $10 \%$ polyacrylamide gels containing $8 \mathrm{M}$ urea. In cases where dideoxy sequencing was done, a clone of actin in Ml3 was used as the template with the actin oligonucleotide as a primer. The sequencing reactions were performed with a kit from Pharmacia.

\section{Acknowledgments}

We thank Drs. D. Horowitz and T.-H. Chang for their critical reading and comments on the manuscript. This work was supported by National Institutes of Health grant GM32637.

\section{References}

Atkinson, K.D. 1985. Two recessive suppressors of Saccharomyces cerevisiae cho1 that are unlinked but fall in the same complementation group. Genetics 777: 1-6.

Bindereif, A. and M.R. Green. 1987. An ordered pathway of snRNP binding during mammalian pre-mRNA splicing complex assembly. EMBO I. 6: 2415-2424.

Brody, E. and J. Abelson. 1985. The 'spliceosome': Yeast premRNA associates with a $40 \mathrm{~S}$ complex in a splicing dependent reaction. Science 228: 963-967.

Chang, T.-H., M.W. Clark, A.J. Lustig, M.E. Cusick, and J. Abelson. 1988. RNA11 protein is associated with yeast spliceosome and is localized in the periphery of the cell nucleus. Mol. Cell. Biol, 8: 2379-2393.

Cheng, S.-C. and J. Abelson. 1986. Fractionation and character- 
ization of yeast mRNA splicing extract. Proc. Natl. Acad. Sci. 83: 2387-2391.

- 1987. Spliceosome assembly in yeast. Genes Dev. 1: $1014-1027$.

Domdey, H., B. Apostol, R.-J. Lin, A.J. Newman, E. Brody, and J. Abelson. 1984. Lariat structures are in vivo intermediates in yeast pre-mRNA splicing. Cell 39: 611-621.

Feinberg, A.P. and B. Vogelstein. 1983. A technique for radiolabeling DNA restriction endonuclease fragments to high specific activity. Anal. Biochem. 132: 6-13.

Frendewey, D. and W. Keller. 1985. Stepwise assembly of premRNA splicing complex requires U-snRNPs and specific intron sequences. Cell 42: 355-367.

Grabowski, P.J., S.R. Seiler, and P.A. Sharp. 1985. A multicomponent complex is involved in splicing of messenger RNA precursors. Cell 42: 345-353.

Green, M.R. 1986. Pre-mRNA splicing. Annu. Rev. Genet. 20: $671-708$.

Guthrie, C. and B. Patterson. 1988. Spliceosomal snRNAs. Annu. Rev. Genet. 22: 387-419.

Hartwell, L.H. 1967. Macromolecular synthesis in temperature-sensitive mutants in yeast. J. Bacteriol. 93: 1662-1670.

Hartwell, L.H., C. McLaughlin, and J. Warner. 1970. Identification of ten genes that control ribosome formation in yeast. Mol. Gen. Genet. 109: 42-56.

Jackson, S.P., M. Lossky, and J.D. Beggs. 1988. Cloning of RNA8 of Saccharomyces cerevisiae, detection of the RNA 8 protein and demonstration that it is essential for nuclear pre-mRNA splicing. Mol. Cell. Biol. 8: 1067-1075.

Konarska, M.M. and P.A. Sharp. 1986. Electrophoretic separation of complexes involved in the formation of spliceosomes. Cell 46: 845-855.

-1987. Interactions between small nuclear ribonuclear particles in the formation of spliceosomes. Cell 49: 763774.

Lamond, A.I., M.M. Konarska, P.J. Grabowski, and P.A. Sharp. 1988. Spliceosome assembly involves the binding and release of U4 small nuclear ribonucleoprotein. Proc. Natl. Acad. Sci. 85: 411-415.

Larkin, J.C and J.L. Woolford. 1983. Molecular cloning and analysis of the CRY1 gene: A highly conserved ribosomal protein gene. Nucleic Acids Res. 11: 403-420.

Last, R.L., J.B. Stavenhagen, and J.L. Woolford. 1984. Isolation and characterization of the RNA2, RNA3, and RNA11 genes of Saccharomyces cerevisiae. Mol. Cell. Biol. 4: 23962405.

Lee, M.G., R.A. Young, and J.D. Beggs. 1984. Cloning of the RNA2 gene of Saccharomyces cerevisiae. EMBO $\%$ 3: $2825-2830$.

Lin, R.-J., A.J. Lustig, and J. Abelson. 1987. Splicing of yeast nuclear pre-mRNA in vitro requires functional $40 \mathrm{~S}$ spliceosomes and several extrinsic factors. Genes Dev. 1: 7-18.

Lossky, M., G.J. Anderson, S.P. Jackson, and J.D. Beggs. 1987. Identification of yeast snRNP protein and the detection of snRNP-snRNP interactions. Cell 51: 1019-1026.

Lustig, A.J., R.-J. Lin, and J. Abelson. 1986. The yeast RNA gene products are essential for mRNA splicing in vitro. Cell 47: 953-963.

Maniatis, T. and R. Reed. 1987. The role of small nuclear ribonucleoproteins in pre-mRNA splicing. Nature 325: 673-678.

Miller, A.M. 1984. The yeast MATa1 gene contains two introns. EMBO J. 3: 1061-1065.

Moir, D., S.E. Stewart, B.C. Osmond, and D. Botstein. 1982. Cold sensitive cell-division-cycle mutants of yeast: Isolation, properties, and pseudoreversion studies. Genetics 100: $547-563$.

$\mathrm{Ng}, \mathrm{R}$. and J. Abelson. 1980. Isolation and sequence of the gene for actin in Saccharomyces cerevisiae. Proc. Natl. Acad. Sci. 77: 3912-3916.

Padgett, R.A., P.J. Grabowski, M.M. Konarska, S. Seiler, and P.A. Sharp. 1986. Splicing of messenger RNA precursors. Annu. Rev. Biochem. 55: 1119-1150.

Parker, R., P.G. Siliciano, and C. Guthrie. 1987. Recognition of the TACTAAC box during mRNA splicing in yeast involves base pairing to U2 like snRNA. Cell 49: 229-239.

Pikielny, C.W. and M. Rosbash. 1985. mRNA splicing efficiency in yeast and the contribution of nonconserved sequences. Cell 41: 119-126.

Pikielny, C.W., B.C. Rymond, and M. Rosbash. 1986. Electrophoresis of ribonucleoproteins reveals an ordered assembly of yeast splicing complexes. Nature 324: $341-345$.

Rosbash, M., P.K. W. Harris, J.L. Woolford, and J.L. Teem. 1981. The effect of temperature sensitive rna mutants on the transcription products from cloned ribosomal protein genes of yeast. Cell 24: 679-686.

Ruby, S.R. and J. Abelson. 1988. An early and heirarchic role for U1 snRNP in spliceosome assembly. Science 242: 10281035.

Ruskin, B., P.D. Zamore, and M.R. Green. 1988. A factor U2AF is required for $\mathrm{U} 2$ snRNP binding and splicing complex assembly. Cell 52: 207-219.

Seraphin, B.L., L. Kretzner, and M. Rosbash. 1988. A U1 snRNA: Pre-mRNA base pairing interaction is required early in yeast spliceosome assembly but does not uniquely define the 5' cleavage site. EMBO f. 7: 2533-2538.

Shapiro, M.B. and P. Senapathy. 1987. RNA splice junction of different classes of eucaryotes: Sequence statistics and functional implications in gene expression. Nucleic Acids Res. 15: $7155-7174$.

Sharp, P.A. 1987. Splicing of messenger RNA precursors. Science 235: 766-771.

Sherman, F., G.R. Fink, and J.B. Hicks. 1986. Methods in yeast genetics. Cold Spring Harbor Laboratory, Cold Spring Harbor, New York.

Soltyk, A., M. Tropak, and J.D. Friesen. 1984. Isolation and characterization of the RNA2, RNA4, and RNA11 genes of Saccharomyces cerevisiae. J. Bacteriol. 160: 1093-1100.

Stearns, T. and D. Botstein. 1988. Unlinked non-complementation: Isolation of new conditional-lethal mutations in each of the tubulin genes of Saccharomyces cerevisiae. Genetics 119: $249-260$.

Steitz, J.A., D.L. Black, V. Gerke, K.A. Parker, A. Kraemer, D. Frendewey, and W. Keller. 1987. Function of the abundant U-snRNPs. In Structure and function of major and minor SNURPS (ed. M. Birnsteil), Springer-Verlag, New York.

Tazi, J., C. Alibert, I. Reveilland, G. Cathala, C. Brunel, and P. Jeanteur. 1986. A protein that specifically recognizes the $3^{\prime}$ splice site of mammalian pre-mRNA introns is associated with a small nuclear ribonucleoprotein. Cell 47: 755-766.

Teem, J.L. and M. Rosbash. 1983. Expression of $\beta$-galacosidase gene containing ribosomal protein 51 intron is sensitive to the rna2 mutation of yeast. Proc. Natl. Acad. Sci. 80: 44034407.

Teem, J.L., J.R. Rodrigues, L. Tung, and M. Rosbash. 1983. The rna2 mutation affects the processing of actin as well as ribosomal protein mRNAs. Mol. Gen. Genet. 192: 101-103.

Vijayraghavan, U. and J. Abelson. 1989. Pre-mRNA splicing in yeast. In Nucleic acids and molecular biology (ed. F. Eckstein, and D.J. Lilley), Springer Verlag. (in press).

Warner, J.R. 1987. Applying genetics to the splicing problem. Genes Dev. 1: 1-3.

Zhuang, Y. and A.M. Weiner. 1986. A compensatory base change in Ul snRNA suppresses a $5^{\prime}$ splice site mutation. Cell 46: 827-835. 


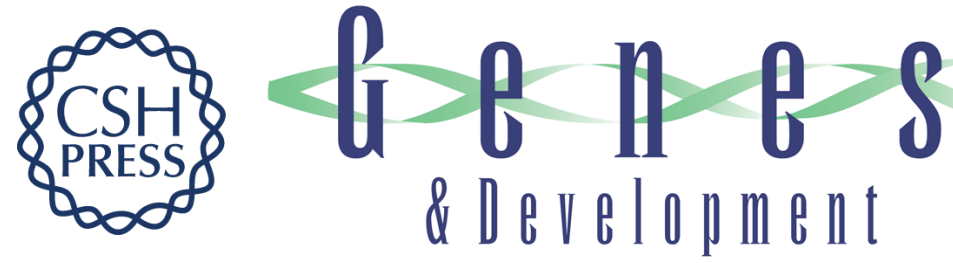

\section{Isolation and characterization of pre-mRNA splicing mutants of Saccharomyces cerevisiae.}

U Vijayraghavan, M Company and J Abelson

Genes Dev. 1989, 3:

Access the most recent version at doi:10.1101/gad.3.8.1206

References This article cites 45 articles, 15 of which can be accessed free at:

http://genesdev.cshlp.org/content/3/8/1206.full.html\#ref-list-1

License

Email Alerting

Service

Receive free email alerts when new articles cite this article - sign up in the box at the top right corner of the article or click here.

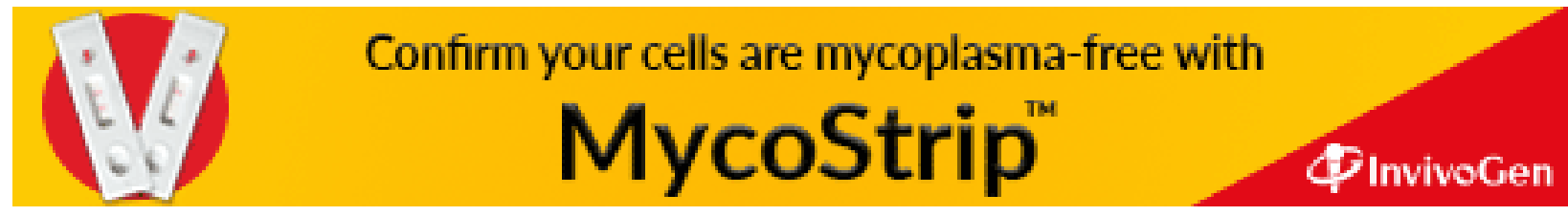

